\title{
Diálogos articuladores del poder en los reinos cristianos medievales
}

Nuria Corral Sánchez

Daniel Justo SÁnchez

Rosa Quetglas Munar

Universidad de Salamanca

Power articulation dialogues in Medieval Iberia 

Las relaciones de poder son un objeto de estudio de gran interés para conocer mejor las sociedades medievales. La investigación en este campo goza de una larga tradición, pero las ópticas desde la que puede enfocarse permiten todavía una gran diversidad. En base a esta premisa, durante el VII International Medieval Meeting de Lleida (2017) presentamos dos mesas redondas en torno al diálogo político en la Península Ibérica desde el siglo VI hasta el fin del periodo medieval, en una visión diacrónica que se expuso de forma comparativa en cada sesión.

La primera, centrada en cuestiones teórico-metodológicas, albergó aportaciones vinculadas a las relaciones políticas en la monarquía visigoda, las corrientes de investigación dedicadas, por un lado, a los castillos como centros de control territorial y símbolos de poder, y, por otro, al papel del discurso y la comunicación en el contexto político bajomedieval castellano. En la segunda, por su parte, fueron presentados algunos estudios de caso, que aportaron perspectivas complementarias sobre el papel de las convenientiae en las dinámicas sociales catalanas del siglo XI, el ejercicio del poder en los castillos gallegos plenomedievales y al liderazgo político en el terreno bélico castellano del siglo XV.

De todas estas aportaciones, por diferentes circunstancias, solo algunas han podido ser recogidas en el monográfico que presentamos hoy en estas páginas. No obstante, consideramos que esta participación supone una muestra representativa del interés sobre estas temá- ticas, insertas en el amplio marco de la historia política, pero desde perspectivas renovadas. Esa creemos que es la principal fortaleza de este número, la aportación de ciertas innovaciones teóricas y metodológicas para el estudio de un área con una extensa trayectoria historiográfica, muchas veces vinculada a ciertas corrientes tradicionalistas.

El primer trabajo que presentamos en este monográfico es el de Pablo Poveda Arias (Universität Hamburg), que ha estudiado el reinado de Amalarico (5II-53I) con el fin de ilustrar las dinámicas de poder vigentes en un periodo germinal del reino visigodo hispano. Se trata de una lectura alejada de la perspectiva teleológica y de los análisis excesivamente negativos que han sido transmitidos por algunas fuentes históricas y sectores de la historiografía moderna. De esta forma, se obtiene un perfil del monarca que subraya el pragmatismo con el que hizo frente a las distintas amenazas internas que marcaron su reinado.

Posteriormente, Daniel Justo Sánchez (Universidad de Salamanca) propone un estado de la cuestión y una reflexión teórica sobre los castillos alto y plenomedievales, en la que integra los postulados creados por las investigaciones sobre el incastellamento italiano y las perspectivas que interpretan estos lugares como símbolos de poder plasmados en el paisaje. El objetivo de este estudio es reflexionar sobre las posibilidades de estas dos vías de análisis en la investigación sobre los castillos del noroeste peninsular, un espacio que, 
aunque ya ha sido objeto de estudios de calidad, todavía poseen potencial de desarrollo, especialmente en el marco de corrientes europeas, de la mano de la arqueología y de las nuevas tecnologías.

Por último, el trabajo de Nuria Corral Sánchez (Universidad de Salamanca), desde un punto de vista cercano a la historia cultural, subraya el valor de la comunicación para el estudio de las relaciones sociopolíticas en general y de los conflictos medievales en particular. En estas páginas, la autora realiza una aproximación teórica sobre las diferentes estrategias discursivas empleadas por los actores políticos en la Edad Media tardía, ofreciendo una propuesta metodológica que aplica al estudio de la contestación política a partir de crónicas y fuentes literarias del siglo $\mathrm{XV}$ castellano.
No queríamos terminar esta breve introducción sin acordarnos de todas las personas que de una forma u otra han contribuido al desarrollo de este monográfico, desde los evaluadores hasta la editora de la revista, Raquel Alonso Álvarez, que amablemente nos ha acompañado en este viaje. Asimismo, no podemos olvidar a los demás compañeros que participaron con nosotros en el congreso de Lleida, así como a los asistentes que enriquecieron esas horas con sus comentarios e ideas. En especial, queremos remarcar la colaboración de Carlos Lixó Gómez (USC) y Ekaitz Etxeberria Gallastegi (UPV) quienes, como ponentes, aportaron interesantes comunicaciones. Al mismo tiempo, agradecemos a Álvaro Carvajal Castro (CSIC) y a Fernando Luis Corral (USAL) que aceptaran desinteresadamente moderar las sesiones en el sofocante calor leridano de finales de junio. 